UUITP-20/09

\title{
Magnon dispersion to four loops in the ABJM and ABJ models
}

\author{
J. A. Minahan ${ }^{1}$, O. Ohlsson Sax ${ }^{1}$ and C. Sieg ${ }^{2}$ \\ ${ }^{1}$ Department of Physics and Astronomy, Uppsala University \\ SE-751 08 Uppsala, Sweden \\ joseph.minahan@fysast.uu.se, olof.ohlsson-sax@physics.uu.se \\ ${ }^{2}$ The Niels Bohr International Academy \\ The Niels Bohr Institute \\ Blegdamsvej 17, DK-2100, Copenhagen Ø, Denmark \\ csieg@nbi.dk
}

\begin{abstract}
The ABJM model is a superconformal Chern-Simons theory with $\mathcal{N}=6$ supersymmetry which is believed to be integrable in the planar limit. However, there is a coupling dependent function that appears in the magnon dispersion relation and the asymptotic Bethe ansatz that is only known to leading order at strong and weak coupling. We compute this function to four loops in perturbation theory by an explicit Feynman diagram calculation for both the ABJM model and the ABJ extension. We find that all coefficients have maximal transcendentality. We then compute the four-loop wrapping correction for a scalar operator in the $\mathbf{2 0}$ of $S U(4)$ and find that it agrees with a recent prediction from the ABJM $Y$-system of Gromov, Kazakov and Vieira. We also propose a limit of the ABJ model that might be perturbatively integrable at all loop orders but has a short range Hamiltonian.
\end{abstract}




\section{Introduction and summary of results}

There are two seemingly different superconformal field theories which have an integrable structure in their planar limit. The first of these is $\mathcal{N}=4$ super Yang-Mills which has been the subject of intense study for more than a decade. The latter is the ABJM model for a superconformal Chern-Simons theory with gauge group $U(N) \times U(N)$, where the first gauge group has a Chern-Simons action at level $k$ and the second has level $-k$ [1]. This theory was only proposed last year as the conjectured holographic dual to $M$-theory on $\mathrm{AdS}_{4} \times \mathrm{S}^{7} / \mathrm{Z}_{k}$.

The integrability of these theories appears in the two point functions of gauge invariant operators. In the $\mathcal{N}=4$ case a class of gauge invariant operators are single traces of $S U(N)$ adjoint fields, whose anomalous dimensions can be found by mapping the problem to a spin chain [2 4]. The spins are in the singleton representation of $P S U(2,2 \mid 4)$, the full superconformal group of $\mathcal{N}=4$ SYM. In the ABJM case, the fundamental fields are in bifundamental representations of $U(N) \times U(N)$ and the corresponding spin chain is of alternating type [5-7]. The spins on the odd sites are in one of the two singleton representations of $O S p(6 \mid 4)$, the full superconformal group of the ABJM model, and the spins on the even sites are in the other singleton representation [8,9].

The spin chains in both theories have ground states that correspond to the chiral primary operators in their respective gauge theories. These are operators whose dimensions are protected by the supersymmetry and thus have zero anomalous dimension. A convenient choice for a spin chain ground state in $\mathcal{N}=4$ is $\operatorname{tr}\left(Z^{L}\right)$, while in the ABJM model a convenient choice is $\operatorname{tr}\left(\left(Y^{1} Y_{4}^{\dagger}\right)^{L}\right)$. The other single trace operators are then constructed by introducing magnons that change the fields in the chain. The magnons themselves transform in a short representation of a subgroup of the superconformal group, which is $S U(2 \mid 2) \ltimes S U(2 \mid 2)$ for $\mathcal{N}=4$ and $S U(2 \mid 2)$ for ABJM.

The presence of the $S U(2 \mid 2)$ structures imposes severe constraints on the magnon dispersion relations. As was shown in [10], this dispersion relation must have the form

$$
E(p)=\sqrt{Q^{2}+4 h^{2}(\lambda) \sin ^{2} \frac{p}{2}}-Q,
$$

where $p$ is the momentum of the magnon on the spin chain and $Q$ is the $R$-charge of the magnon. For $\mathcal{N}=4$ the charge for a fundamental magnon is $Q=1$, while in ABJM the charge is $Q=\frac{1}{2} \cdot h^{2}(\lambda)$ is a function of the 't Hooft coupling $\lambda$, which in $\mathcal{N}=4$ is $\lambda=g_{\mathrm{YM}}^{2} N$, while in ABJM it is $\lambda=N / k$. Moreover, the 't Hooft coupling only enters the asymptotic Bethe equations through this same function $h^{2}(\lambda)$ [11].

To the best of our knowledge, integrability makes no prediction for $h^{2}(\lambda)$, but there exist some alternative analyses not based on integrability [12,13]. Thus, it is important to understand its origin and how it plays a role in the AdS/CFT correspondence. In the $\mathcal{N}=4$ case, all known results in perturbation theory and from the study of the string theory dual are consistent with $h^{2}(\lambda)=\lambda /\left(4 \pi^{2}\right)$. However, in the ABJM model it is known at the level of two-loop perturbation theory that $h^{2}(\lambda)=\lambda^{2}+\mathrm{O}\left(\lambda^{4}\right)$ [5, 6, 14, while at large coupling we know by taking the BMN limit of type IIA string theory on $\mathrm{AdS}_{4} \times \mathrm{CP}^{3}$ [6, 15] and from one-loop string corrections] [16] that $h^{2}(\lambda)=\frac{1}{2} \lambda-\frac{\ln 2}{\sqrt{2} \pi} \sqrt{\lambda}+$ $\mathrm{O}(1)$. In fact, since the perturbative expansion is even in $\lambda$, the function $h^{2}(\lambda)$ should have a square root branch cut along the negative real axis.

\footnotetext{
${ }^{1}$ We thank Arkady Tseytlin for comments on this.
} 
The calculation we present here also considers the ABJ modification of the ABJM model [17], where the gauge group is generalized to $U(M) \times U(N)$, but with the levels of the gauge group kept at $k$ and $-k$. In this case there are now two 't Hooft couplings defined as

$$
\lambda=\frac{M}{k}, \quad \hat{\lambda}=\frac{N}{k},
$$

but the superconformal group is still $O S p(6 \mid 4)$. It was shown at the two-loop level in the scalar sector [18] and in the full $O S p(6 \mid 4)$ sector [9] that $\lambda^{2}$ is replaced by $\lambda \hat{\lambda}$, but otherwise the dilatation operator is the same, and is therefore still integrable 2 If in the planar limit the integrability is to persist to higher loop orders, then the only modification that can occur is in the function $h^{2}$. In general it will be a two parameter function, $h^{2}(\bar{\lambda}, \sigma)$, where we define $\bar{\lambda}$ and $\sigma$ as

$$
\bar{\lambda}=\sqrt{\lambda \hat{\lambda}}, \quad \sigma=\frac{\lambda-\hat{\lambda}}{\bar{\lambda}} .
$$

Since the number of colors is different, parity is explicitly broken in the ABJ model. Therefore, one might expect, for example, the odd site magnons would have different dispersion relations from the even site magnons, or that $h^{2}(\bar{\lambda}, \sigma) \neq h^{2}(\bar{\lambda},-\sigma)$. This breaking has indeed been observed beyond the planar limit [20]. However, in the planar limit this does not seem to be the case. Instead, there is still a parity symmetry at the level of the planar diagrams that prevents either of these from occurring. Nonetheless, we still find some interesting behaviour.

Up to four loops, the expansion of $h^{2}(\bar{\lambda}, \sigma)$ assumes the following form

$$
h^{2}(\bar{\lambda}, \sigma)=\bar{\lambda}^{2}+\bar{\lambda}^{4} h_{4}(\sigma), \quad h_{4}(\sigma)=h_{4}+\sigma^{2} h_{4, \sigma}
$$

where $h_{4}$ is the same constant as in the ABJM case, in which $\sigma=0$. In this paper we determine $h_{4}(\sigma)$ by computing the four-loop contribution to the dilatation operator. We find that the coefficients in (1.4) are explicitly 3

$$
h_{4}=-4 \zeta(2) \approx-6.58, \quad h_{4, \sigma}=-\zeta(2)
$$

The negative sign for $h_{4}$ is sensible, as this will dampen the quadratic behaviour found at small $\bar{\lambda}$ to the linear behaviour at large $\bar{\lambda}$. Interestingly, $h_{4}$ has no rational contributions, leaving a maximally transcendental result. It is also striking that the coefficients in (1.5) are integers.

In order to find $h_{4}(\sigma)$, it was necessary to compute the contributions of several dozen Feynman diagrams. The diagrams were evaluated using dimensional reduction [21]. All diagrams started in three dimensions, where there appear three dimensional $\epsilon$-tensors from the gauge propagators in the Chern-Simons Lagrangian and from parity violating fermion terms. However, all terms carried an even number of $\epsilon$-tensors which could be contracted with each other, leaving metric tensors only. Once the integrands were in this form, the integrals were dimensionally regularized, reducing the dimension to $D=3-2 \varepsilon$.

\footnotetext{
${ }^{2}$ In the ABJM case, hints for a breakdown of integrability beyond the planar limit have explicitly been found in [19].

3 A previous version of this paper had a different value of $h_{4, \sigma}$ because of an incorrect coupling dependence of $S_{5}$ in equation (3.6). This change only concerns the result in the more general ABJ case.
} 
The underlying loop integrals were computed using scalar $G$-functions, integration by parts relations [22,23] and generalizations thereof. Special care had to be taken due to the presence of infrared divergences, which in three dimensions arise whenever there are three scalar propagators attached to a vertex with no external momentum entering the vertex. As checks for the results for the integrals, we verified relations between integrals and recalculated certain integrals using GPXT [24,25] and also certain transformations between two-dimensional master integrals [26].

The result for $h_{4}$ in (1.5) is strikingly similar to the leading wrapping correction for length four operators, which has been obtained by Gromov, Kazakov and Vieira [27] by means of the proposed $Y$-system [28 30] for the ABJM model 4 In this paper we explicitly compute the wrapping correction with our methods and find agreement with their result.

In section 2 we give further details on the relation of $h^{2}(\bar{\lambda}, \sigma)$ to the dilatation operator. In section 3 we discuss the necessary Feynman diagrams and list their values. A more explicit description of our methods will be given in a later publication [33]. In section 4 we collect our results from the previous section and compute $h_{4}(\sigma)$. We also discuss an interesting limit of the ABJ model where one finds an integrable Hamiltonian with next-to-nearest neighbour interactions. In section 5 we compute the wrapping corrections for the length four operator in the $\mathbf{2 0}$ representation of $S U(4)$. In section 6 we present our conclusions and suggest further lines of inquiry. There are also three appendices that contain further technical details.

\section{Extraction of $h^{2}(\bar{\lambda}, \sigma)$}

To compute the coefficients $h_{4}$ and $h_{4, \sigma}$ in a field theory calculation, it is only necessary to consider a single scalar magnon. Thus, we may restrict our study to the $S U(2)$ sector of the full $O S p(6 \mid 4)$ group. Actually, we will enlarge this to the $S U(2) \times S U(2)$ sector. Inside the $S U(2) \times S U(2)$ sector, the magnons in the first $S U(2)$ live on the odd sites, while those in the other $S U(2)$ live on the even sites. The two different types of magnons do not interact with each other until the six-loop level [11] so for our purposes we may treat them as noninteracting.

The dispersion relation (1.1) yields the following expansion in a power series in $\bar{\lambda}$

$$
E(p)=2 \bar{\lambda}^{2}(1-\cos p)+2 \bar{\lambda}^{4}\left(h_{4}(\sigma)-3+\left(4-h_{4}(\sigma)\right) \cos p-\cos 2 p\right)+\mathcal{O}\left(\bar{\lambda}^{6}\right),
$$

where we have set $Q=1 / 2$ and we have reexpressed powers of $\sin \frac{p}{2}$ in terms of cosine. The exponentials which are contained in the cosine functions are powers of $e^{i p}$, which is the eigenvalue of the shift operator that moves the magnon over by two sites on the spin chain. Hence, at the four-loop level there is a maximal shifting of 4 sites, which does not depend on $h_{4}$. However, for shifts of 2 sites, there is an $h_{4}$ dependence.

An ansatz for the dilatation operator which eigenvalues are $E(p)$ can be constructed by considering the (open) single magnon momentum / shift operator eigenstate

$$
\psi_{p}=\sum_{k=0}^{L} e^{i p k}\left(Y^{1} Y_{4}^{\dagger}\right)^{k} Y^{2} Y_{4}^{\dagger}\left(Y^{1} Y_{4}^{\dagger}\right)^{L-k-1}
$$

\footnotetext{
${ }^{4}$ This result is not altered by a correction [31,32] of the originally proposed $Y$-system.
} 
The momentum dependence in the expansion (2.1) is a result of acting with certain products of permutations on the above state, where a permutation exchanges the fields between two nearest neighbour either odd or even sites. Similar to the $\mathcal{N}=4 \mathrm{SYM}$ case, we introduce the permutation structures

$$
\left\{a_{1}, a_{2}, \ldots, a_{m}\right\}=\sum_{i=1}^{L} \mathrm{P}_{2 i+a_{1} 2 i+a_{1}+2} \mathrm{P}_{2 i+a_{2} 2 i+a_{2}+2} \ldots \mathrm{P}_{2 i+a_{m}} 2 i+a_{m}+2
$$

where we identify $L+i \simeq i$ when we act on a cyclic state of length $L$. Some details about these structures and the permutation basis at four loops can be found in appendix B. Up to four loops, the dilatation operator expands as

$$
D=L+\bar{\lambda}^{2} D_{2}+\bar{\lambda}^{4} D_{4}(\sigma)+\mathcal{O}\left(\bar{\lambda}^{6}\right)
$$

At these orders, $D_{k}, k=2,4$ decomposes into a direct sum $D_{k}=D_{k \text {,odd }}+D_{k \text {,even }}$ of two operators, that act non-trivially on either odd or even sites only. Since up to this order the magnon dispersion relations for odd and even sites are identical, the operators for even and odd sites have identical coefficients and read

$$
\begin{aligned}
D_{2, \text { even }} & =\{\}-\{1\}, \\
D_{2, \text { odd }} & =\{\}-\{2\}, \\
D_{4, \text { odd }}(\sigma) & =\left(h_{4}(\sigma)-4\right)\{\}+\left(6-h_{4}(\sigma)\right)\{1\}-\{1,3\}-\{3,1\}, \\
D_{4, \text { even }}(\sigma) & =\left(h_{4}(\sigma)-4\right)\{\}+\left(6-h_{4}(\sigma)\right)\{2\}-\{2,4\}-\{4,2\} .
\end{aligned}
$$

The above expressions are easily obtained by considering the action of the permutation structures for odd sites on the state (2.2). Neglecting boundary effect, we find

$$
\{\} \rightarrow 1, \quad\{1\} \rightarrow \mathrm{e}^{i p}+\mathrm{e}^{-i p}, \quad\{1,3\} \rightarrow \mathrm{e}^{2 i p}+2 \mathrm{e}^{-i p}, \quad\{3,1\} \rightarrow \mathrm{e}^{-2 i p}+2 \mathrm{e}^{i p} .
$$

Comparing the resulting expression with (2.1) then fixes the coefficients of the non-trivial permutations in $D_{4, \text { odd }}$ in terms of $h_{4}(\sigma)$. The coefficient of the identity \{\} is then found by demanding that the cyclic ground state has eigenvalue zero. Since the dispersion relations for magnons on odd and even sides are identical, the same coefficients are found for $D_{4, \text { even }}$.

Notice that in (2.5) there is no term that mixes odd and even sites, i.e. which contains the permutation structure $\{1,2\}$. It could in principle appear in a four site interaction, but explicitly cancels [34].

\section{Four-loop calculation}

In the following we list in brief the sets of logarithmically divergent diagrams which have to be considered to compute the asymptotic four-loop dilatation operator $D_{4}$ in the $S U(2) \times S U(2)$ subsector. The composite operators in this sector do not contain subtraces. We therefore can neglect all diagrams which only contribute to the coefficient of the trace operator. Furthermore the contribution of the identity can be inferred from supersymmetry. We can hence reconstruct $D_{4}$ in the $S U(2) \times S U(2)$ by only considering the diagrams which contribute to flavour permutations, and we drop all contributions 
to the trace and identity operator in the result. This simplification is indicated by an arrow pointing from the diagram to the respective result.

In all four-loop diagrams, the first leg is attached to an odd site, i.e. to a field $Y^{i}$ of the composite operator (denoted by a bold line). The color loop in the lower left corner of each diagram will hence contribute a factor $M$ to the diagram. The elementary scalar, fermion gauge and ghost fields are denoted by respectively plain, dashed, wiggly and dotted lines. The action from which the Feynman rules are extracted is given in appendix A.

\subsection{Substructures}

Before turning to the four-loop diagrams, we introduce fixed combinations of substructures which appear in the four-loop diagrams. These substructures themselves do not lead to non-trivial flavour exchanges. Besides these structures, a four-loop diagram which contributes to the coefficients of non-trivial permutations in the dilatation operator (2.5) then has to contain a scalar six vertex. Power counting then restricts the substructures to the ones shown below.

Flavour-neutral next-to-nearest neighbour interactions can only be realized with gauge boson exchange and at least two cubic gauge scalar vertices at the first and last leg. The relevant diagrams involve the structure

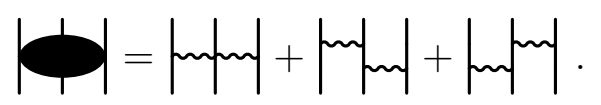

Flavour-neutral nearest-neighbour interactions involve the following diagrams

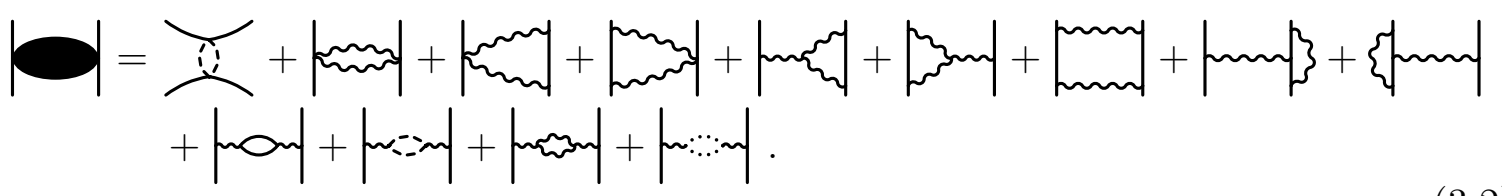

In the first term, which contains a fermion loop, the flavour flows from the upper to the respective lower external line as in all the other diagrams.

The two-loop self-energy correction of the scalar field also appears as a sum of subdiagrams. In terms of the non-vanishing diagrams it explicitly reads

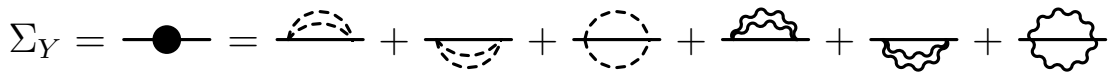

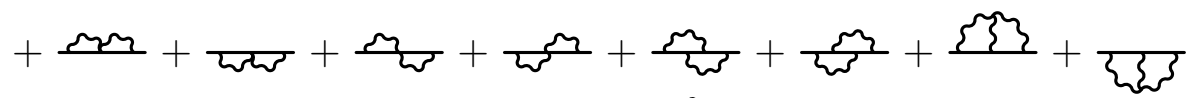

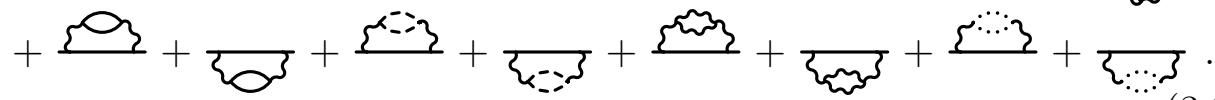

To calculate the divergences of the corresponding four-loop diagrams, we need the divergent and finite parts of the two-loop self-energy. Apart from the Wick rotation (the result has to be multiplied by a factor $i^{2}=-1$ ), the amputated scalar self-energy contribution becomes

$$
\begin{aligned}
\Sigma_{Y}=-1[ & \frac{\lambda \hat{\lambda}}{4}\left(\frac{3}{2 \varepsilon}-\frac{3}{2} \pi^{2}+3\left(\frac{25}{3}-\gamma+\ln 4 \pi\right)\right) \\
& \left.+\frac{(\lambda-\hat{\lambda})^{2}}{4}\left(\frac{1}{4 \varepsilon}-\frac{\pi^{2}}{4}+\frac{1}{2}(3-\gamma+\ln 4 \pi)\right)\right] \underline{-1+2 \varepsilon}
\end{aligned}
$$


where the last propagator factor on the r.h.s. captures the momentum dependence. Its weight label indicates the exponent of $\frac{1}{p^{2}}$, where $p$ is the external momentum. The pole part of the above result coincides with the result in [18].

The amputated fermionic one-loop self energy is given by

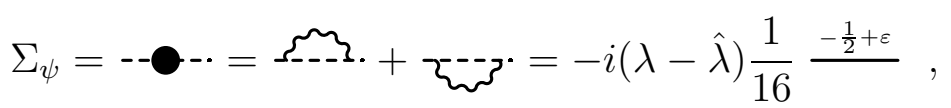

where the left and right fermion fields respectively are $\psi$ and $\psi^{\dagger}$.

\subsection{Diagrams involving only the scalar six-vertex}

The diagrams which lead to non-trivial flavour permutations are given by

$$
S_{2}=\frac{(\lambda \hat{\lambda})^{2}}{16}\left(-\frac{1}{2 \varepsilon^{2}}+\frac{2}{\varepsilon}\right)(\{1,3\}-\{1\})
$$

As explained at the beginning of this section, we have neglected all contributions to the trace and identity operator. We have also not evaluated the remaining scalar diagrams

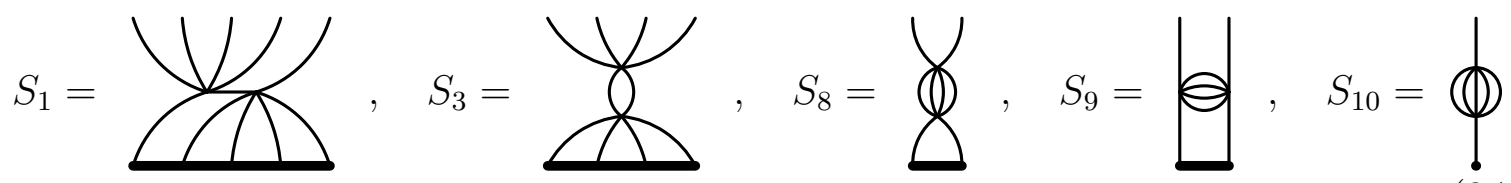

which only contribute to the trace or identity operator.

Along with the set of diagrams in (3.6), we also need the reflected diagrams of $S_{2}$, $S_{4}, S_{7}$, which are easily obtained by using the transformations in appendix C. From the diagram $S_{5}$ and from its shifted version, in which $\lambda$ and $\hat{\lambda}$ are interchanged, we have to consider the terms involving the permutation $\{1\}$. The sum of the relevant set of 
diagrams with two scalar six-vertices then reads

$$
\begin{aligned}
S=\frac{\lambda \hat{\lambda}}{16}[ & \lambda \hat{\lambda}\left(\left(-\frac{1}{2 \varepsilon^{2}}+\frac{2}{\varepsilon}\right)(\{1,3\}+\{3,1\}+2\{1,2\})+\left(\frac{4}{\varepsilon^{2}}-\frac{1}{\varepsilon}\left(12-\frac{1}{4} \pi^{2}\right)\right)\{1\}\right) \\
& \left.+(\lambda-\hat{\lambda})^{2} \frac{\pi^{2}}{8 \varepsilon}\{1\}\right] .
\end{aligned}
$$

\subsection{Diagrams involving a fermion square}

The only four-loop diagrams in which four neighboured scalar lines interact, and which contain a fermion square loop are given by

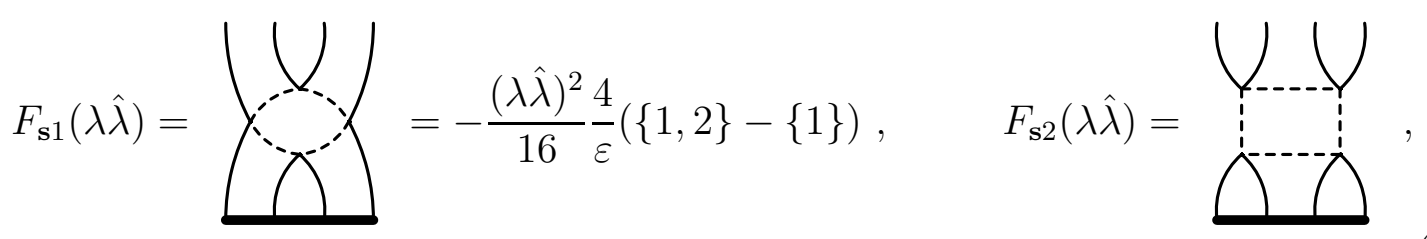

where the second diagram only contributes to the identity and trace part of the dilatation operator and hence is not considered here.

Besides the above diagrams, there are the following diagrams in which only three neighbouring lines interact. They contain either a bubble and are given by

$$
\begin{aligned}
& F_{\mathbf{s b} 1}(\lambda \hat{\lambda})=\overbrace{\mathbf{s b} 2}(\lambda \hat{\lambda})=-\frac{(\lambda \hat{\lambda})^{2}}{16} \frac{1}{\varepsilon^{2}}\{1\}, \quad
\end{aligned}
$$

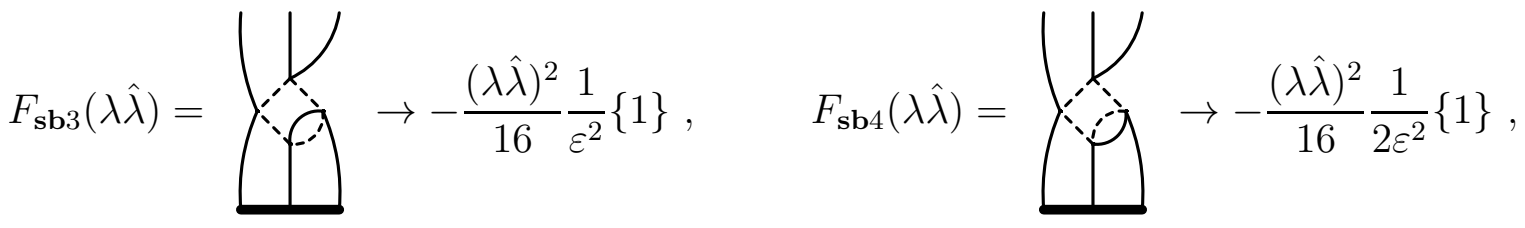

$$
\begin{aligned}
& F_{\mathbf{s b} 5}(\lambda \hat{\lambda})=\longleftarrow-\frac{(\lambda \hat{\lambda})^{2}}{16} \frac{1}{\varepsilon^{2}}\{1\}, \quad F_{\mathbf{s b} 6}(\lambda \hat{\lambda})=
\end{aligned}
$$

or they contain triangles and read

$$
F_{\text {st } 1}(\lambda \hat{\lambda})=
$$


Summing up the above contributions, allowing for factors of two that come from reflections of the diagrams $F_{\text {sb1 }}(\lambda \hat{\lambda})$ to $F_{\text {sb } 6}(\lambda \hat{\lambda})$ and $F_{\text {st } 2}(\lambda \hat{\lambda})$, we obtain

$$
F_{\mathbf{s}}=\frac{(\lambda \hat{\lambda})^{2}}{16}\left[-\frac{4}{\varepsilon}\{1,2\}+\left(-\frac{6}{\varepsilon^{2}}+\frac{2}{3 \varepsilon} \pi^{2}\right)\{1\}\right] .
$$

\subsection{Diagrams involving fermion triangles}

The four-loop diagrams in which three quartic scalar fermion vertices form a fermion triangle also involve a single gluon propagator. The relevant diagrams are given by

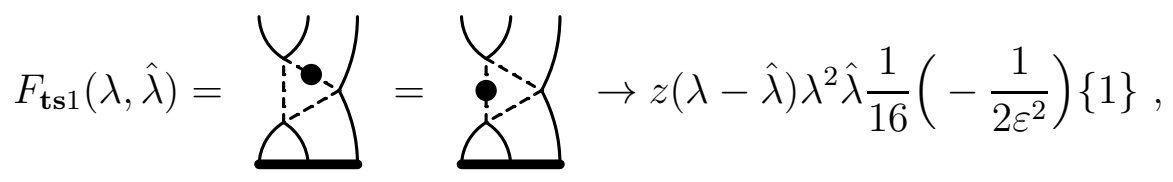

$$
\begin{aligned}
& F_{\mathrm{ts} 2}(\lambda, \hat{\lambda})=\overbrace{-}^{-} \rightarrow z(\lambda-\hat{\lambda}) \lambda^{2} \hat{\lambda} \frac{1}{16}\left(-\frac{1}{2 \varepsilon^{2}}+\frac{2}{\varepsilon}\right)\{1\}
\end{aligned}
$$

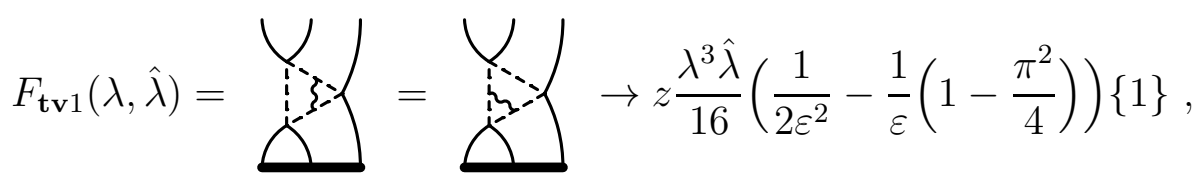

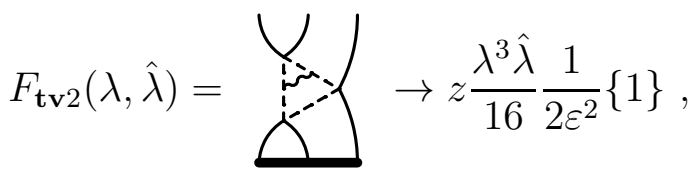

$$
\begin{aligned}
& F_{\mathrm{tv} 3}(\lambda \hat{\lambda})=\left\{\begin{array}{c}
(\lambda \hat{\lambda})^{2} \\
16
\end{array}\right. \\
& F_{\mathrm{tv} 4}(\lambda \hat{\lambda})=\bigcup_{-1}^{-2} \rightarrow z \frac{(\lambda \hat{\lambda})^{2}}{16} \frac{1}{\varepsilon}\left(3-\frac{\pi^{2}}{4}\right)\{1\} \\
& F_{\mathbf{t v} 5}(\lambda \hat{\lambda})=\underbrace{}_{1}=\frac{(\lambda \hat{\lambda})^{2}}{16} \frac{1}{\varepsilon}\left(-1+\frac{\pi^{2}}{4}\right)\{1\} .
\end{aligned}
$$

The result depends on a sign $z= \pm 1$ which is not uniquely given in the literature [7,35]. We will fix $z$ by computing the renormalization of the scalar six vertex at two loops. It should vanish for the correct sign choice of $z$ to ensure superconformal invariance.

Considering also the reflected diagrams, constructed by using the transformation rules of appendix $\mathbb{C}$, we obtain for the sum of the above diagrams

$$
F_{\mathbf{t}}=z \frac{\lambda \hat{\lambda}}{16}\left[\lambda \hat{\lambda}\left(\frac{3}{\varepsilon^{2}}+\frac{1}{\varepsilon}\left(12-\frac{\pi^{2}}{3}\right)\right)+(\lambda-\hat{\lambda})^{2} \frac{\pi^{2}}{2 \varepsilon}\right]\{1\} .
$$

The presence of the reflected diagrams which do not differ by a sign from the original diagram, but only by an exchange $\lambda \leftrightarrow \hat{\lambda}$ thereby guarantees that the result only depends quadratically on the difference of the couplings and hence on the parameter $\sigma$ defined in (1.3). 


\subsection{Diagrams involving a single scalar six-vertex and flavour- neutral substructures}

The two-loop diagram involving a single scalar six-vertex is promoted to logarithmically divergent four-loop diagrams by adding appropriate flavour-neutral substructures. We find diagrams which are completed by the next-to-nearest or nearest neighbour interactions given in (3.1) and (3.2) or by the two-loop self energy correction of the scalar field (3.3). Keeping only the contributions to the non-trivial permutation, we find

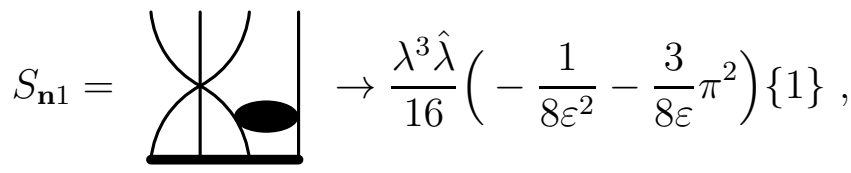

$$
\begin{aligned}
& S_{\mathbf{n} 2}=\rightarrow \frac{\lambda^{3} \hat{\lambda}}{16}\left(-\frac{1}{8 \varepsilon^{2}}+\frac{1}{2 \varepsilon}\right)\{1\}, \\
& S_{\mathbf{v} 1}=\frac{(\lambda \hat{\lambda})^{2}}{16} \frac{1}{\varepsilon}\left(2-\frac{\pi^{2}}{6}\right)\{1\} \text {, }
\end{aligned}
$$

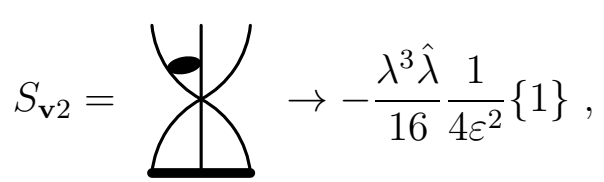

$$
\begin{aligned}
& S_{\mathbf{v} 3}=\rightarrow \frac{\lambda \hat{\lambda}^{3}}{16}\left(-\frac{1}{8 \varepsilon^{2}}-\frac{3 \pi^{2}}{8 \varepsilon}\right)\{1\}, \\
& S_{\mathbf{v} 4}=\bigotimes_{16}\left[\lambda \hat{\lambda} \frac{1}{\varepsilon}\left(8-\frac{2}{3} \pi^{2}\right)+\lambda^{2}\left(-\frac{1}{4 \varepsilon^{2}}+\frac{1}{\varepsilon}\left(1-\frac{7}{12} \pi^{2}\right)\right)\right]\{1\} \\
& S_{\mathbf{s} 1}=\frac{\lambda \hat{\lambda}}{16}\left[\lambda \hat{\lambda} \frac{3}{2 \varepsilon^{2}}+(\lambda-\hat{\lambda})^{2} \frac{1}{4 \varepsilon^{2}}\right]\{1\} \\
& S_{\mathbf{s} 2}=\bigcup \rightarrow \frac{\lambda \hat{\lambda}}{16}\left[\lambda \hat{\lambda}\left(\frac{3}{4 \varepsilon^{2}}-\frac{1}{\varepsilon}\left(11-\frac{3}{4} \pi^{2}\right)\right)+(\lambda-\hat{\lambda})^{2}\left(\frac{1}{8 \varepsilon^{2}}-\frac{1}{\varepsilon}\left(\frac{1}{2}-\frac{1}{8} \pi^{2}\right)\right)\right] \underset{(3.15)}{\{1\}} \text {. }
\end{aligned}
$$

The reflected diagrams for the first two diagrams are constructed according to the transformations in appendix C. The diagrams $S_{\mathbf{v} 1}$ to $S_{\mathbf{v} 4}$ then contribute with a factor of two. The diagrams $S_{\mathbf{s} 1}$ contributes with a factor $\frac{3}{2}$, since there are three external fields which can obtain a self-energy correction which counts with factor $\frac{1}{2}$ in the renormalization of the composite operator. Finally, $S_{\mathbf{s} 2}$ contributes with a factor of three. We find for the sum

$$
S_{\mathbf{n}}=\frac{\lambda \hat{\lambda}}{16}\left[\lambda \hat{\lambda}\left(\frac{11}{4 \varepsilon^{2}}-\frac{1}{\varepsilon}\left(12+\frac{23}{12} \pi^{2}\right)\right)+(\lambda-\hat{\lambda})^{2}\left(-\frac{1}{8 \varepsilon^{2}}-\frac{23}{24 \varepsilon} \pi^{2}\right)\right]\{1\} .
$$




\subsection{Two-loop renormalization of the scalar six-vertex}

The two-loop vertex renormalization of the scalar six-vertex allows us to fix a sign discrepancy in the literatur 5 parameterized by $z= \pm 1$ which affects the four-loop diagrams with fermion triangles, c.f. (3.13) and (3.14). Neglecting a factor $i^{2}=-1$ for the Wick rotation, the renormalization of the six-scalar vertex is found as

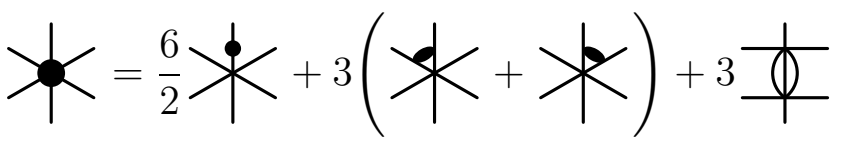

$$
\begin{aligned}
& +3(\text { - } \\
& =\lambda \hat{\lambda} \frac{3}{2 \varepsilon} i(z-1)\{1\} \text {. }
\end{aligned}
$$

Non-renormalization of the coupling and hence superconformal invariance requires $z=1$, which corresponds to the sign choice in [35].

\section{Result}

The four-loop term of the renormalization constant of the composite operators

$$
\mathcal{O}_{a, \text { ren }}=\mathcal{Z}_{a}{ }^{b} \mathcal{O}_{b, \text { bare }}, \quad \mathcal{Z}=\mathbb{1}+\bar{\lambda}^{2} \mathcal{Z}_{2}+\bar{\lambda}^{4} \mathcal{Z}_{4}+\ldots
$$

is given as the negative of the sum of $S, F_{\mathbf{s}}, F_{\mathbf{t}}, S_{\mathbf{n}}$ in equations (3.8), (3.12), (3.14), (3.16). Fixing $z=1$ as determined in subsection 3.6, we obtain

$$
\begin{aligned}
\mathcal{Z}_{4} \rightarrow \frac{1}{16} & {\left[\left(\frac{1}{2 \varepsilon^{2}}-\frac{2}{\varepsilon}\right)(\{1,3\}+\{3,1\})+\frac{1}{\varepsilon^{2}}\{1,2\}\right.} \\
& \left.+\left(-\frac{15}{4 \varepsilon^{2}}+\frac{1}{\varepsilon}\left(12+\frac{4}{3} \pi^{2}\right)+\sigma^{2}\left(\frac{1}{8 \varepsilon^{2}}+\frac{\pi^{2}}{3 \varepsilon}\right)\right)\{1\}\right] .
\end{aligned}
$$

The dilatation operator is then obtained from the renormalization constant $\mathcal{Z}$ as

$$
D=\lim _{\varepsilon \rightarrow 0}\left[2 \varepsilon \bar{\lambda} \frac{\mathrm{d}}{\mathrm{d} \bar{\lambda}} \ln \mathcal{Z}(\bar{\lambda}, \varepsilon)\right] .
$$

Effectively, the above definition extracts the coefficient of the $\frac{1}{\varepsilon}$ pole at a given loop order $L$ and multiplies it by a factor $2 L$. The four-loop dilatation operator for odd sites is thus the coefficient of the $\frac{1}{\varepsilon}$ pole of (4.2) multiplied by 8 . We must still add the neglected identity part, which we fix by demanding that the ground state has zero eigenvalue. We thus obtain

$$
D_{4, \text { odd }}=-\left(4+4 \zeta(2)+\sigma^{2} \zeta(2)\right)\{\}+\left(6+4 \zeta(2)+\sigma^{2} \zeta(2)\right)\{1\}-\{1,3\}-\{3,1\},
$$

where $\zeta(2)=\frac{\pi^{2}}{6}$. By comparing this result with (2.5)), we immediately find that the four-loop term of the function $h^{2}(\lambda)$ in (1.4) is given by

$$
h_{4}(\sigma)=-4 \zeta(2)-\sigma^{2} \zeta(2) .
$$

\footnotetext{
${ }^{5}$ The antisymmetric parts in the product of two $\gamma$ matrices (A.1) in [35] and [7] differ by a sign.
} 


\subsection{An interesting limit}

The presence of the extra parameter $\sigma$ suggests an interesting limit. Suppose that $\lambda \gg \hat{\lambda}$ while $\lambda$ is still in the perturbative regime. This corresponds to a dominance of the term $\sigma^{2} h_{4, \sigma}$ w.r.t. $h_{4}$ at four loops. Letting $\mathcal{D}=\hat{\lambda}^{-1}(D-2 L)$, then $\mathcal{D}$ in this limit reduces to

$$
\mathcal{D}=\lambda\left(1-\zeta(2) \lambda^{2}\right)(2\{\}-\{1\}-\{2\})
$$

for the $S U(2) \times S U(2)$ sector. In other words, $\mathcal{D}$ is the Hamiltonian for two Heisenberg spin chains! In fact, at higher loops, it is clear that the dominant contribution also comes from the next-to-nearest neighbour interactions with the same permutation structure, hence in this limit $\mathcal{D}$ is the same local Hamiltonian as in (4.6), except with the $\lambda$ dependent prefactor replaced with an all-loop function $f(\lambda)$.

Whether or not the integrability holds outside of the $S U(2) \times S U(2)$ sector to four loops or higher remains to be seen. It is also true that this scaling only applies to the anomalous dimension and not to the full dimension of the operator. Added to this, there are reasons to believe that the ABJ Chern-Simons theory is inconsistent if $\lambda-\hat{\lambda}>1$ [17], thus this limit is likely to fail at strong coupling.

\section{$5 \quad$ Four-loop wrapping interactions}

To find the correct anomalous dimensions of a length four state at four loops, we have to consider the wrapping interactions [36,37]. Recently, Gromov, Kazakov and Vieira have made a prediction for the four-loop wrapping contribution for scalar operators in the $\mathbf{2 0}$ representation of $S U(4)$ [27]. The highest weight of this representation is in the $S U(2) \times$ $S U(2)$ sector. These authors find their result by applying the thermodynamic Bethe ansatz (TBA), first introduced for the original AdS/CFT correspondence in [38, 39], using the predicted asymptotic Bethe equations [11]. In particular, based on the string hypothesis for the mirror theory [40], the TBA is formulated in terms of a $Y$-system [28 32], a series of difference equations, that lend themselves to an efficient order by order solution.

Following the strategy of [41, we first subtract from the asymptotic dilatation operator (2.5) the range five interactions (given by $S_{2}$ and the reflected diagram). This leaves for $D_{4, \text { odd }}$ in (4.4)

$$
D_{4, \text { odd }}^{\text {sub }}=\left(4+4 \zeta(2)+\sigma^{2} \zeta(2)\right)(\{1\}-\{\}) .
$$

We then have to add the following wrapping diagrams which contain a permutation 
structure

$$
W_{1}=\frac{(\lambda \hat{\lambda})^{2}}{16}\left(-\frac{1}{2 \varepsilon^{2}}+\frac{2}{\varepsilon}\right)(\{1,3\}-\{1\})
$$

We then sum the above diagrams, where we include a factor of two for $W_{3}$ since its reflection cannot be mapped to itself by a cyclic rotation. To this sum we add the identity terms needed to give zero wrapping for a chiral primary, remembering that $\{1,3\}$ is equivalent to \{\} for length four operators. Taking the negative of the sum, extracting the residues of $\frac{1}{\epsilon}$ and multiplying by 8 as in the previous section, we find that the wrapping contribution to the odd site dilatation operator is given by

$$
D_{4, \text { odd }}^{\mathrm{w}}=-(4-2 \zeta(2))(\{1\}-\{\}) .
$$

The 20 in the $S U(2) \times S U(2)$ sector is a completely antisymmetric state, so the eigenvalue of $\{1\}-\{\}$ is -8 . Hence the four-loop wrapping contribution to the anomalous dimension is

$$
\gamma_{20}^{\mathrm{w}}=(32-16 \zeta(2)) \bar{\lambda}^{4},
$$

which agrees with the GKV $Y$-system prediction [27]. It does not depend on $\sigma^{2}$, which is expected from the topology of the diagrams and the fact that the two-loop result does not depend on $\sigma^{2}$. Adding $D_{4, \text { odd }}^{\text {sub }}$ to $D_{4, \text { odd }}^{\mathrm{w}}$, we find that the anomalous dimension of the $\mathbf{2 0}$ is given by

$$
\gamma_{\mathbf{2 0}}=4+8 \bar{\lambda}^{2}-\left(48 \zeta(2)+8 \sigma^{2} \zeta(2)\right) \bar{\lambda}^{4},
$$

where we have included the lower orders [5]. It is striking that the rational parts coming from wrapping terms cancel the rational contribution in (5.1), leaving a maximally transcendental four-loop result for $\gamma_{20}$. 


\section{Discussion}

In this paper we computed the four-loop correction to $h^{2}(\bar{\lambda}, \sigma)$. The coefficients $h_{4}$ and $h_{4, \sigma}$ both have maximal transcendentality. The significance of this is hard to say. It would be interesting to see if this pattern continues to higher loops.

In section 2 we argued that the perturbative expansion is invariant under parity to four-loop order in the coupling. Assuming that our arguments can be extended to higher loops, $h^{2}(\bar{\lambda}, \sigma)$ would then have a perturbative expansion in even powers of $\sigma$. On the string world-sheet a nonzero $\sigma$ comes from turning on a $b$ field flux through the 2-cycle of $\mathrm{CP}^{3}$ [17]. This leads to nonperturbative corrections that do not directly affect the strong coupling behavior of $h^{2}(\bar{\lambda}, \sigma)$. However, there is a change in the radius of $\mathrm{AdS}_{4}$ and $\mathrm{CP}^{3}$, which in the scaling limit is $[17,42] 6$

$$
R_{s}^{2}=2^{5 / 2} \pi \sqrt{\min (\lambda, \hat{\lambda})-\frac{1}{24}+\frac{1}{2}(\lambda-\hat{\lambda})^{2}},
$$

where $\min (\lambda, \hat{\lambda})$ is the minimum of $\lambda$ and $\hat{\lambda}$. Hence, for $\bar{\lambda} \gg 1 h^{2}(\bar{\lambda}, \sigma)$ has a cut in the $\sigma^{2}$ plane with a branch point at $\sigma^{2}=0$. To do a parity transformation, we could smoothly deform $\sigma$ to $-\sigma$. If we circle the branch point then $h^{2}$ is not invariant and parity would seem to be broken. From the string theory analysis in [17] it seems that the correct thing to do is not to circle the branch point, keeping parity invariant. It would be helpful to better understand what the physical implications are of going to the other sheet.

We have also proposed a new local spin chain that arises when $\lambda \gg \hat{\lambda}$. If this limit is integrable, then the Hamiltonian is guaranteed by the $\mathcal{N}=6$ supersymmetry to have the form in [8,9], only differing by an overall function of $\lambda$. It would be very interesting to check if it can be extended outside the $S U(2) \times S U(2)$ sector or to higher loops. A feasible test would be in the scalar $S U(4)$ sector where integrability requires certain relative coefficients between nontrivial terms in the Hamiltonian.

It has been argued that the $U(M)_{k} \times U(N)_{-k}$ theory is nonunitary if $M>N+k$ [17], which corresponds to $\lambda-\hat{\lambda}>1$. A possible signal of this in our limiting local spin chain would be the Hamiltonian changing sign at $\lambda=1$. If this were to happen, then the non BPS operators would have negative anomalous dimensions, a clear violation of unitarity. Since the sign of $h_{4, \sigma}$ is negative, this seems reasonably plausible.

One can also speculate on why $h^{2}(\bar{\lambda}, \sigma)$ is more complicated for $\mathrm{ABJ}(\mathrm{M})$ than for $\mathcal{N}=$ 4 SYM. A possible clue is that $U(M)_{k} \times U(N)_{-k}$ is believed to be equivalent to $U(N)_{k} \times$ $U(2 N-M+k)_{-k}$ for $M>N$ [17]. In terms of $\lambda$ and $\hat{\lambda}$ this corresponds to equivalence under $\lambda \rightarrow \hat{\lambda}, \hat{\lambda} \rightarrow 2 \hat{\lambda}-\lambda+1$, which translates into rather complicated transformations for $\bar{\lambda}$ and $\sigma$. If the ABJ theory is integrable, then under this transformation $h^{2}(\bar{\lambda}, \sigma)$ must be invariant. From this alone we see that $h^{2}$ has to be a rather complicated function of both $\bar{\lambda}$ and $\sigma$. Perhaps the asymptotic information for $h^{2}(\bar{\lambda}, \sigma)$ and the invariance under these transformations will be enough to ultimately find the complete function.

We have also verified the GKV prediction for the wrapping correction to the $\mathbf{2 0}$. This serves as an important check for the $Y$-system conjecture and also provides a

${ }^{6}$ There could be a further correction to $R_{s}$ because of a half-integer shift of the $b$ field flux through the 2-cycle of $\mathrm{CP}^{3}$ [4]. We thank O. Bergman for discussions on this. 
useful consistency check for us. Finally, we note that as $h_{4}(\lambda)$ also the first wrapping correction is maximally transcendental.

\section{Acknowledgments}

We would like to thank O. Bergman, T. Klose and K. Zarembo for very helpful discussions. The research of J. A. M. is supported in part by the Swedish research council. The research of C. S. is supported in part by the European Marie Curie Research and Training Network ENRAGE (MRTN-CT-2004-005616). J. A. M. thanks the CTP at MIT and the Galileo Institute in Florence for kind hospitality during the course of this work. Support for these visits comes from the STINT foundation and INFN. O. O. S. thanks NBI and C. S. thanks Uppsala University for hospitality during the course of this work.

\section{A Conventions}

In three dimensional spacetime with metric $\eta_{\mu \nu}=\operatorname{diag}(-,+,+)$ The antisymmetric tensor is normalized as $\epsilon^{012}=1$. The product of $\gamma$-matrices is given by

$$
\gamma_{\mu} \gamma_{\nu}=\eta_{\mu \nu}+z \epsilon_{\mu \nu \rho} \gamma^{\rho}
$$

where $z= \pm 1$ represents a sign. The Clifford algebra is given by

$$
\left\{\gamma^{\mu}, \gamma^{\nu}\right\}=2 \eta^{\mu \nu}\left(\begin{array}{ll}
1 & 0 \\
0 & 1
\end{array}\right) \text {. }
$$

The action reads

$$
\begin{aligned}
S_{\mathrm{kin}}=\frac{k}{4 \pi} \int \mathrm{d} \mathrm{d}^{3} x \operatorname{tr}[ & A_{\alpha}\left(\epsilon^{\alpha \beta \gamma} \partial_{\beta}-\frac{1}{\zeta} \partial^{\alpha} \partial^{\gamma}\right) A_{\gamma}-\hat{A}_{\alpha}\left(\epsilon^{\alpha \beta \gamma} \partial_{\beta}-\frac{1}{\zeta} \partial^{\alpha} \partial^{\gamma}\right) \hat{A}_{\gamma} \\
& \left.+Y_{A}^{\dagger} \partial_{\mu} \partial^{\mu} Y^{A}+i \psi^{\dagger B} \not \partial \psi_{B}+c^{*} \partial_{\mu} \partial^{\mu} c+\hat{c}^{*} \partial_{\mu} \partial^{\mu} \hat{c}\right] \\
S_{\mathrm{int}}=\frac{k}{4 \pi} \int \mathrm{d}^{3} x \operatorname{tr}[ & \frac{2}{3} i \epsilon^{\alpha \beta \gamma}\left(A_{\alpha} A_{\beta} A_{\gamma}-\hat{A}_{\alpha} \hat{A}_{\beta} \hat{A}_{\gamma}\right) \\
& -i A_{\mu} Y^{A} \stackrel{\leftrightarrow}{\partial^{\mu}} Y_{A}^{\dagger}-i \hat{A}_{\mu} Y_{A}^{\dagger} \dot{\partial}^{\mu} Y^{A}+2 Y_{A}^{\dagger} A_{\mu} Y^{A} \hat{A}^{\mu} \\
& -\hat{A}_{\mu} \hat{A}_{\mu} Y_{A}^{\dagger} Y^{A}-A_{\mu} A_{\mu} Y^{A} Y_{A}^{\dagger} \\
& -\psi^{\dagger B} A \psi_{B}+\hat{A}_{\mu} \psi^{\dagger B} \gamma^{\mu} \psi_{B}-i A^{\mu}\left[c, \partial_{\mu} c^{*}\right]-i \hat{A}^{\mu}\left[\hat{c}, \partial_{\mu} \hat{c}^{*}\right] \\
& +\frac{1}{12} Y^{A} Y_{B}^{\dagger} Y^{C} Y_{D}^{\dagger} Y^{E} Y_{F}^{\dagger}\left(\delta_{A}^{B} \delta_{C}^{D} \delta_{E}^{F}+\delta_{A}^{F} \delta_{C}^{B} \delta_{E}^{D}-6 \delta_{A}^{B} \delta_{C}^{F} \delta_{E}^{D}+4 \delta_{A}^{D} \delta_{C}^{F} \delta_{E}^{B}\right) \\
& -\frac{i}{2}\left(Y_{A}^{\dagger} Y^{B} \psi^{\dagger C} \psi_{D}-\psi_{D} \psi^{\dagger C} Y^{B} Y_{A}^{\dagger}\right)\left(\delta_{B}^{A} \delta_{C}^{D}-2 \delta_{C}^{A} \delta_{B}^{D}\right) \\
& \left.+\frac{i}{2} \epsilon^{A B C D} Y_{A}^{\dagger} \psi_{B} Y_{C}^{\dagger} \psi_{D}-\frac{i}{2} \epsilon_{A B C D} Y^{A} \psi^{\dagger B} Y^{C} \psi^{\dagger D}\right]
\end{aligned}
$$

The Feynman rules are extracted from this action. 


\section{B Permutation structures}

Since the operators in the $S U(2) \times S U(2)$ subsector are free of subtraces, the corresponding dilatation operator is given by an expansion in terms of the permutation structures defined in (2.3).

The definition is similar as in the $\mathcal{N}=4$ SYM case, [3], but each permutation in the product permutes neighbouring fields at either odd or even sites. Also, the permutation structures are shifted by two sides then inserted along the chain and not by one site as in the $\mathcal{N}=4 \mathrm{SYM}$ case. Permutations at even and odd insertion points commute with each other, and only do not commute if they have one position in common, i.e. if the integers in the argument lists of the permutation structures differ by two. The rules for manipulation which have to be modified compared to the ones in the $\mathcal{N}=4 \mathrm{SYM}$ case are

$$
\begin{aligned}
\{\ldots, a, b, \ldots\} & =\{\ldots, b, a, \ldots\}, \quad|a-b| \neq 2, \\
\{a, \ldots, b\} & =\{a+2 n, \ldots, b+2 n\} .
\end{aligned}
$$

We can shift all odd integers to the left and all even integers in the argument lists to the right. The basis of permutation structures at four loops hence reads

$$
\{\},\{1\},\{2\},\{1,2\},\{3,2\},\{1,3\},\{3,1\}, \quad\{2,4\}, \quad\{4,2\} .
$$

The dilatation operator decomposes into three parts, acting on even, on odd and on mixed sides, respectively. These parts are defined by containing permutation structures with respectively only odd, only even and both odd and even arguments.

\section{Symmetries}

Let $c(\lambda, \hat{\lambda})\left\{a_{1}, \ldots, a_{m}\right\}$ be the result associated with a certain Feynman graph. We can easily construct the contribution from the analogous graph shifted by one step along the chain of elementary fields from the corresponding operator. This exchanges all fields as

$$
A \leftrightarrow \hat{A}, \quad Y \leftrightarrow Y^{\dagger}, \quad \psi \leftrightarrow \psi^{\dagger}, \quad c \leftrightarrow \hat{c}, \quad c^{\star} \leftrightarrow \hat{c}^{\star}
$$

and it exchanges the colour loops of both gauge groups, i.e. it exchanges $\lambda \leftrightarrow \hat{\lambda}$. We also have to consider several sign changes due to the exchange of certain vertices and of the propagators of the two gauge fields. By $P_{x}$ and $V_{x}$ we denote the multiplicities with which the corresponding propagator or vertex of type $x$ appears in the graph. A shift by one side then transforms a Feynman graph as

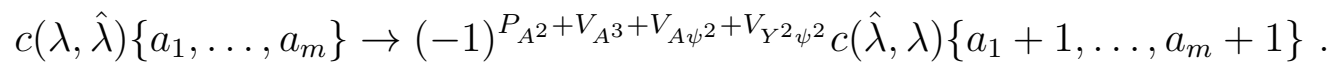

Furthermore, the reflection of a given non reflection-symmetric Feynman graph contributes to the perturbation series. A reflection exchanges $A \leftrightarrow \hat{A}$ and it changes also the sign of all loop momenta. A reflection hence transforms a Feynman graph as

$$
c(\lambda, \hat{\lambda})\left\{a_{1}, \ldots, a_{m}\right\} \rightarrow(-1)^{P_{A^{2}}+V_{A Y^{2}}+V_{A \psi^{2}}+V_{Y^{2}} \psi^{2}} c(\hat{\lambda}, \lambda)\left\{-a_{1}, \ldots,-a_{m}\right\} .
$$




\section{References}

[1] O. Aharony, O. Bergman, D. L. Jafferis, and J. Maldacena, $\mathcal{N}=6$ superconformal Chern-Simons-matter theories, M2-branes and their gravity duals, arXiv:0806.1218.

[2] J. A. Minahan and K. Zarembo, The Bethe-ansatz for $\mathcal{N}=4$ super Yang-Mills, JHEP 0303 (2003) 013, hep-th/0212208.

[3] N. Beisert, C. Kristjansen, and M. Staudacher, The dilatation operator of $\mathcal{N}=4$ conformal super Yang-Mills theory, Nucl. Phys. B664 (2003) 131-184, hep-th/0303060.

[4] N. Beisert and M. Staudacher, The $\mathcal{N}=4 S Y M$ Integrable Super Spin Chain, Nucl. Phys. B670 (2003) 439-463, hep-th/0307042.

[5] J. A. Minahan and K. Zarembo, The Bethe ansatz for superconformal Chern-Simons, JHEP 09 (2008) 040, arXiv:0806.3951.

[6] D. Gaiotto, S. Giombi, and X. Yin, Spin Chains in $\mathcal{N}=6$ Superconformal Chern-Simons-Matter Theory, arXiv:0806.4589.

[7] D. Bak and S.-J. Rey, Integrable Spin Chain in Superconformal Chern-Simons Theory, JHEP 10 (2008) 053, arXiv:0807.2063.

[8] B. I. Zwiebel, Two-loop Integrability of Planar $\mathcal{N}=6$ Superconformal Chern-Simons Theory, arXiv:0901.0411.

[9] J. A. Minahan, W. Schulgin, and K. Zarembo, Two loop integrability for Chern-Simons theories with $\mathcal{N}=6$ supersymmetry, JHEP 03 (2009) 057, arXiv:0901.1142.

[10] N. Beisert, The su(2|2) dynamic S-matrix, hep-th/0511082.

[11] N. Gromov and P. Vieira, The all loop AdS4/CFT3 Bethe ansatz, arXiv:0807.0777.

[12] D. Berenstein and D. Trancanelli, Three-dimensional $\mathcal{N}=6$ SCFT's and their membrane dynamics, Phys. Rev. D78 (2008) 106009, arXiv:0808.2503.

[13] D. Berenstein and D. Trancanelli, S-duality and the giant magnon dispersion relation, arXiv:0904.0444

[14] G. Grignani, T. Harmark, and M. Orselli, The $S U(2) \times S U(2)$ sector in the string dual of $\mathcal{N}=6$ superconformal Chern-Simons theory, arXiv:0806.4959.

[15] T. Nishioka and T. Takayanagi, On Type IIA Penrose Limit and $\mathcal{N}=6$ Chern-Simons Theories, JHEP 08 (2008) 001, arXiv:0806.3391.

[16] T. McLoughlin, R. Roiban, and A. A. Tseytlin, Quantum spinning strings in $A d S_{4} \times C P^{3}$ : testing the Bethe Ansatz proposal, JHEP 11 (2008) 069, arXiv:0809.4038.

[17] O. Aharony, O. Bergman, and D. L. Jafferis, Fractional M2-branes, JHEP 11 (2008) 043, arXiv:0807.4924.

[18] D. Bak, D. Gang, and S.-J. Rey, Integrable Spin Chain of Superconformal $U(M) \times U(N)$ Chern-Simons Theory, JHEP 10 (2008) 038, arXiv:0808.0170.

[19] C. Kristjansen, M. Orselli, and K. Zoubos, Non-planar ABJM Theory and Integrability, JHEP 03 (2009) 037, arXiv:0811.2150.

[20] P. Caputa, C. Kristjansen, and K. Zoubos, Non-planar ABJ Theory and Parity, Phys. Lett. B677 (2009) 197-202, arXiv:0903.3354.

[21] W. Chen, G. W. Semenoff, and Y.-S. Wu, Two loop analysis of nonAbelian Chern-Simons theory, Phys. Rev. D46 (1992) 5521-5539, hep-th/9209005.

[22] D. I. Kazakov, Calculation of Feynman integrals by the method of 'uniqueness', Theor. Math. Phys. 58 (1984) 223-230.

[23] D. J. Broadhurst, Evaluation of a class of Feynman diagrams for all numbers of loops and dimensions, Phys. Lett. B164 (1985) 356. 
[24] K. G. Chetyrkin, A. L. Kataev, and F. V. Tkachov, New Approach to Evaluation of Multiloop Feynman Integrals: The Gegenbauer Polynomial x Space Technique, Nucl. Phys. B174 (1980) 345-377.

[25] A. V. Kotikov, The Gegenbauer Polynomial Technique: the evaluation of a class of Feynman diagrams, Phys. Lett. B375 (1996) 240-248, hep-ph/9512270.

[26] A. N. Vasiliev, Y. M. Pismak, and Y. R. Khonkonen, 1/N Expansion: calculation of the exponents eta and nu in the order $1 / N^{* * 2}$ for arbitrary number of dimensions, Theor. Math. Phys. 47 (1981) 465-475.

[27] N. Gromov, V. Kazakov, and P. Vieira, Integrability for the Full Spectrum of Planar AdS/CFT, arXiv:0901.3753.

[28] N. Gromov, V. Kazakov, A. Kozak, and P. Vieira, Integrability for the Full Spectrum of Planar AdS/CFT II, arXiv:0902.4458.

[29] D. Bombardelli, D. Fioravanti, and R. Tateo, Thermodynamic Bethe Ansatz for planar AdS/CFT: a proposal, J. Phys. A42 (2009) 375401, arXiv:0902.3930.

[30] G. Arutyunov and S. Frolov, Thermodynamic Bethe Ansatz for the $A d S_{5} \times S^{5}$ Mirror Model, JHEP 05 (2009) 068, [arXiv:0903.0141].

[31] D. Bombardelli, D. Fioravanti, and R. Tateo, TBA and $Y$-system for planar $A d S_{4} / C F T_{3}, N u c l$. Phys. B834 (2010) 543-561, [arXiv:0912.4715].

[32] N. Gromov and F. Levkovich-Maslyuk, Y-system, TBA and Quasi-Classical Strings in AdS4 $\times$ CP3, arXiv:0912.4911.

[33] J. A. Minahan, O. Ohlsson Sax, and C. Sieg, Anomalous dimensions at four loops in N=6 superconformal Chern-Simons theories, arXiv:0912.3460

[34] D. Bak, H. Min, and S.-J. Rey, Generalized Dynamical Spin Chain and 4-Loop Integrability in $\mathcal{N}=6$ Superconformal Chern-Simons Theory, arXiv:0904.4677.

[35] M. Benna, I. Klebanov, T. Klose, and M. Smedback, Superconformal Chern-Simons Theories and $A d S_{4} / C F T_{3}$ Correspondence, JHEP 09 (2008) 072, arXiv:0806.1519.

[36] N. Beisert, V. Dippel, and M. Staudacher, A novel long range spin chain and planar $\mathcal{N}=4$ super Yang-Mills, JHEP 07 (2004) 075, hep-th/0405001.

[37] C. Sieg and A. Torrielli, Wrapping interactions and the genus expansion of the 2- point function of composite operators, Nucl. Phys. B723 (2005) 3-32, hep-th/0505071].

[38] J. Ambjorn, R. A. Janik, and C. Kristjansen, Wrapping interactions and a new source of corrections to the spin-chain / string duality, Nucl. Phys. B736 (2006) 288-301, hep-th/0510171.

[39] G. Arutyunov and S. Frolov, On String S-matrix, Bound States and TBA, JHEP 12 (2007) 024, arXiv:0710.1568.

[40] G. Arutyunov and S. Frolov, String hypothesis for the $A d S_{5} \times S^{5}$ mirror, JHEP 03 (2009) 152, arXiv:0901.1417.

[41] F. Fiamberti, A. Santambrogio, C. Sieg, and D. Zanon, Anomalous dimension with wrapping at four loops in $\mathcal{N}=4$ SYM, Nucl. Phys. B805 (2008) 231-266, arXiv:0806.2095.

[42] O. Bergman and S. Hirano, Anomalous radius shift in AdS(4)/CFT(3), JHEP 07 (2009) 016, arXiv:0902.1743.

[43] O. Aharony, A. Hashimoto, S. Hirano, and P. Ouyang, D-brane Charges in Gravitational Duals of 2+1 Dimensional Gauge Theories and Duality Cascades, arXiv:0906.2390. 